\title{
Pathologic Stage IIIB Cutaneous Melanoma AJCC v8
}

National Cancer Institute

\section{Source}

National Cancer Institute. Pathologic Stage IIIB Cutaneous Melanoma A/CC V8. NCI

Thesaurus. Code C137672.

Stage IIIB includes: (T0, N1b, N1c, M0); (T1a/b-T2a, N1b/c or N2b, M0); (T2b/T3a, N1aN2b, M0). TO: No evidence of primary tumor (e.g., unknown primary or completely regressed melanoma). Thickness: Not applicable. Ulceration status: Not applicable. T1a: Tumor measuring less than $0.8 \mathrm{~mm}$ in thickness. Ulceration status: Without ulceration. T1 b: Tumor measuring less than $0.8 \mathrm{~mm}$ in thickness with ulceration, or 0.8-1.0 $\mathrm{mm}$ with or without ulceration. T2a: Tumor measuring more than 1.0 and equal to or less than 2.0 $\mathrm{mm}$ in thickness. Ulceration status: Without ulceration. T2b: Tumor measuring more than 1.0 and equal to or less than $2.0 \mathrm{~mm}$ in thickness. Ulceration status: With ulceration. T3a: Tumor measuring more than 2.0 and equal to or less than $4.0 \mathrm{~mm}$ in thickness. Ulceration status: Without ulceration. N1b: One clinically detected nodal metastasis. Presence of in-transit, satellite, and/or microsatellite metastases: No. N1c: No regional lymph node disease. Presence of in-transit, satellite, and/or microsatellite metastases: Yes. N2b: Two or three nodal metastases at least one of which was clinically detected. Presence of in-transit, satellite, and/or microsatellite metastases: No. N1a: One clinically occult nodal metastasis (i.e., detected by sentinel lymph node biopsy). Presence of intransit, satellite, and/or microsatellite metastases: No. N2a: T wo or three clinically occult nodal metastases (i.e., detected by sentinel lymph node biopsy). Presence of in-transit, satellite, and/or microsatellite metastases: No. M0: No evidence of distant metastasis. LDH level is not applicable. (AJCC 8th ed.) 